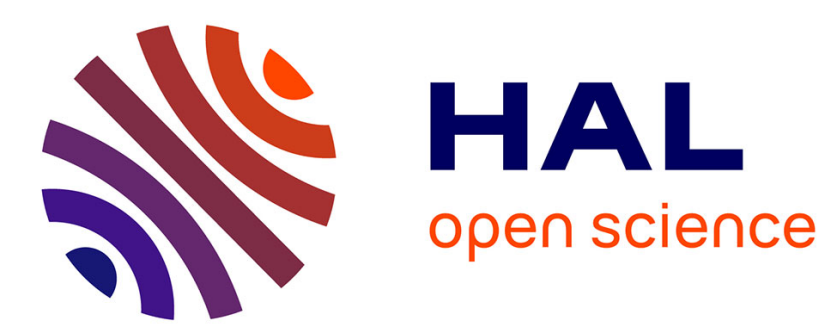

\title{
Towards Complete and Realistic Studies for Rare-Gas Ionic Clusters, the Ar+3 Example
}

Florent X. Gadéa, Mohand Amarouche

\section{To cite this version:}

Florent X. Gadéa, Mohand Amarouche. Towards Complete and Realistic Studies for RareGas Ionic Clusters, the Ar+3 Example. Journal de Physique II, 1995, 5 (12), pp.1767-1776. 10.1051/jp2:1995212 . jpa-00248268

\section{HAL Id: jpa-00248268 https://hal.science/jpa-00248268}

Submitted on 1 Jan 1995

HAL is a multi-disciplinary open access archive for the deposit and dissemination of scientific research documents, whether they are published or not. The documents may come from teaching and research institutions in France or abroad, or from public or private research centers.
L'archive ouverte pluridisciplinaire HAL, est destinée au dépôt et à la diffusion de documents scientifiques de niveau recherche, publiés ou non, émanant des établissements d'enseignement et de recherche français ou étrangers, des laboratoires publics ou privés. 


\title{
Classification
}

Physics Abstracts

$36.40+\mathrm{d}-03.65 \mathrm{Sq}$

\section{Towards Complete and Realistic Studies for Rare-Gas Ionic Clusters, the $\mathrm{Ar}_{3}^{+}$Example}

\author{
Florent Xavier Gadea and Mohand Amarouche(*) \\ Laboratoire de Physique Quantique, IRSAMC, Universite Paul Sabatier, 118 rte de Narbonne \\ 31062 Toulouse Cedex, France
}

(Received 21 April 1995, accepted in final form 14 September 1995)

\begin{abstract}
The approach we have developped for the $a b$ initio simulation of rare-gas ionic clusters is reviewed and applied to $\mathrm{Ar}_{3}^{+}$. It is based on a DIM (Diatomic In Molecules) modelling for the electronic Hamiltonian and transition dipole moments, on approximate estimations for the absorption spectrum and a HWD (Hemiquantal dynamics with the Whole DIM basis) treatment of the fragmentation dynamics. The theoretical study is devoted to three basic aspects: the structure of the ions, their absorption spectrum and their fragmentation dynamics. These aspects correspond respectively to equilibrium, short time and long time dynamics. The results for $\mathrm{Ar}_{3}^{+}$are reviewed (structure, visible spectrum, photofragmentation dynamics, kinetic energy distribution of photofragments) and in addition new ones for the UV absorption spectrum are presented and discussed.
\end{abstract}

\section{Introduction}

Clusters offer the possibility to bridge the gap between molecular and condensed phase properties, simply by increasing the cluster size [1,2]. They represent a challenge for theory which should afford to handle detailed molecular as well as the averaged solid state descriptions, with a unique approach since the evolution is continuous. The usual $a b$-initio techniques, fruitful for the small clusters, become rapidly prohibitive particularly if one is interested in complete studies involving both the electronics (potential energy surfaces) and the dynamics of the system. On the other hand, approximations like tight binding approaches, which are valid for the solid since they take benefit of symmetries and averages other large particle numbers, may become poor ones for the description of small clusters.

The positively ionized rare gas cluster are of particular interest in this context because they can be easily produced and selected. A large amount of experimental results [3-13] concerning their formation (ionization from the neutrals), stabilities, photoabsorption and photofragmentation properties exists. For a theoretical understanding of these results, structural as well as

$\left({ }^{*}\right)$ Permanent address: Laboratoire de Physique et de Chimie Quantique, Université de Tizi Ouzou, Algérie 
dynamical aspects are needed.

One of the most important question in this context is how the initial energy (ionization, photon, collision,...) is absorbed and relaxed in the clisters.

Recently, we have carried out a detailed study [14 20] of the $\mathrm{Ar}_{3}^{+}$ion. The aim of this study was twofold:

i) to address various specific physical problems such as: structure, absorption spectra, photodissociation branching ratio, photofragmentation, kinetic energy distribution,...

ii) to develop the methods in order to perform similar realistic studies, including both the electronic and the dynamical aspects, for larger clusters.

Our approach is only developed from the first principles (ab-initio) and combines three levels of theory:

- ab initio calculation for the initial diatomic data

- modelling of the system with DIM Hamiltonians and modelling of properties like the transition dipole moments

- dynamics involving together the nuclear degrees of freedom (often treated classically) and many electronic states (here all the electronic basis set states).

This approach may have thus a predictive character. The objectives are very similar to the Ab Initio Molecular Dynamics (AIMD) approaches [21 23]. However AIMD is actually focussed on the ground electronic state and is based on SCF approaches while we are investigating physical process which involve various electronic states and have adopted a DIM like modelling. DIM approaches are usually considered as semi-empirical methods. However, it should be emphasized that we use only ab initio data in the DIM approach and that the semi empirical character of our modelling made not here reference to empirical adjustments of parameters. In fact the DIM approach using ab initio effective Hamiltonians inputs leads [24] to a very powerful modelling of the electronic Hamiltonian of large systems. This modelling is able to treat ground as well as excited electronic states. It is particularly suitable for the $\mathrm{Rg}_{n}^{+}$clusters because the electronic basis set can be reduce to the various possible location of the charge (i.e. $3 n$ for Ar to $\mathrm{Xe}, n$ for $\mathrm{He}$ ) and the effective integrals are easily extracted from the dimer calculation. Moreover, the accuracy of this approach for $\mathrm{Rg}_{n}^{+}$is nowadays well established. In the $\mathrm{Rg}_{n}{ }^{+}$ clusters, the charge is localized in a chromophoric core which is usually $\mathrm{Rg}_{3}+[6,25,26,30]$. These clusters can be seen as an ionic core solved by neutral atoms. Therefore the trimer cluster deserves a special attention.

In this paper, the results of our earlier studies are reviewed and some new ones presented. In Section 2, we consider the structure of $\mathrm{Ar}_{3}^{+}$which is of paramount importance for the dynamics. In Section 3 its absorption spectra in the visible and UV range will be presented while in Section 4 its photofragmentation dynamics will be considered. These sections correspond respectively to equilibrium without dynamics (structure of the molecule and analysis of the electronic interactions and of the shape of the potential energy surfaces). short time dynamics (photoabsorption spectra) and long time dynamics (fragmentation).

\section{Structure of $\mathrm{Ar}_{3}^{+}$}

If it is clear that for the dimer the charge is delocalized on the two atoms (in a monoelectronic picture the ground state corresponds to a hole in the highest occupied antibonding orbital) the situation for the trimer is more complex. Because of the directionality of the $3 \mathrm{p}$ orbitals where the hole is mainly localized, the trimer is linear. But there is now a competition between two structures: a symmetry brolien geometry where an ionic dimer polarizes a neutral atom and a symmetric geometry where the charge is delocalized on the tree atoms. The discussion can be 
focussed on the two quantities which play a crucial role, the polarizability of the neutral atoms and the electronic resonance between the two equivalents dimeric ion forms.

The difficulty for the theoretical $a b$ initio prediction is that these two effects appear at different levels of the calculation. The former is present from the SCF level and is mainly sensitive to the basis set quality, while the second needs CI treatments and is rather sensitive to the CI quality level adopted. Therefore ab initio calculations give contradictory results. At the SCF and MP2 level, the localization of the charge dominates [10], however this localization is overestimated and at least POL CI approaches are needed to recover the correct symmetric geometry [27] found by various multireference CI methods [19,28] and also by a recent MP4 calculation [29].

Recently a large CI ab initio calculation has been performed [19] including more than thousand selected determinants for the zeroth order wave function description, $4 \times 10^{4}$ selected determinants whose contribution to the energy were calculated variationnally and about $10^{9}$ determinants for the second order perturbative energy estimation. This calculation has revealed that the linear symmetric geometry is a true minimum of the ground state potential energy surface and that the molecule is more compact than expected. This indicates that the dominant interactions are the resonant ones which forces a complete delocalization of the charge on the three atoms.

Interestingly, the DIM approaches $[15,25,30]$ which use effective integrals extracted from the dimer ion for the resonant interactions, all lead to a correct symmetric result for $\mathrm{Ar}_{3}^{+}$. For the larger Rgen clusters ions only DIM and SCF approaches are possible. The structural results for $\mathrm{Ar}_{3}^{+}$clearly argue in favor of the DIM methods. In addition, as we will see later, the DIM approach do not furnish here only a good estimation of the ground state potential energy surface, but also a complete modelling of the electronic interactions. This idea was at the origin of the HWD method and of a global strategy for realistic studies of the rare gas ions [31].

\section{Absorption Spectrum}

We have developped various approaches for the calculation of the $\mathrm{Ar}_{3}^{+}$absorption spectrum. In time independent methods the absorption cross section is given by the usual golden rule like relation (in atomic units)

$$
\sigma_{i j}(E)=\left(4 \pi^{2} / 3 c\right) E\left|<\psi_{j}\left(E+E_{0}, Q\right)\right| \mu_{i j}(Q)\left|\psi_{i, v}(Q)>\right|^{2}
$$

where $i$ and $j$ label the initial and final electronic state, $E$ is the transition energy (i.e. the photon energy) and $E_{0}$ the ground state vibrational energy, $\psi_{i, v}(Q)$ is the initial (bound) vibrational wave function and $\psi_{j}\left(E+E_{0}, Q\right)$ the final (continuum) one. The evaluation of this integral requires three basic quantities: the ground state vibrational wave function, the electronic transition dipole moment and the excited state vibrational wave function, the approximation of which will generate the various methods.

In our first calculation of the visible absorption spectrum [32], the continuum wave function $\psi_{j}\left(E+E_{0}, Q\right)$ was approximate by the $\delta\left(Q-Q^{\prime}\right)$ function centered in $Q^{\prime}$ such that $E+E_{0}=V_{\mathrm{f}}\left(Q^{\prime}\right), V_{\mathrm{f}}\left(Q^{\prime}\right)$ being the excited electronic state potential. Each geometry $Q$ contributes to the absorption spectrum at the energy $E$ such that $V_{\mathrm{f}}(Q)=E+E_{0}$ by an amount of $\left(4 \pi^{2} / 3 c\right) E \mu_{i j}(Q)^{2} W_{i}(Q)^{2}$, where $W_{i}(Q)^{2}$ is the weight of this geometry in the ground state vibrational wave function. Therefore vertical transitions were assumed at each geometry and $W_{i}(Q)^{2}$ was taken as the ground state $(v=0)$ wave function amplitude $\psi_{i, 0}(Q)^{2}$, for which a product of $1 \mathrm{D}$ harmonic normal mode wave function was assumed: 


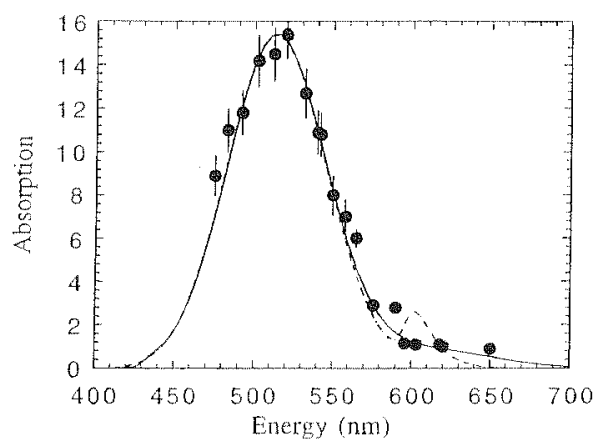

Fig. 1. - Visible absorption spectrum for $\mathrm{Ar}_{3}^{+}$, full line: exact 3D quantum calculation (from Ref. [20]), dashed line: estimation using a convolution of 1D spectra (from Ref. [15]), dots with error bars: experimental spectrum of reference [4]. For a better comparison of the shape, the maximum of the experimental spectrum has been normalized to the theoretical result and its position has been shifted by $10 \mathrm{~nm}$.

$W_{i}(Q)^{2}=\phi_{0}\left(Q_{\mathrm{a}}\right)^{2} \phi_{0}\left(Q_{\mathrm{b}}\right)^{2} \phi_{0}\left(Q_{\mathrm{s}}\right)^{2}, Q_{\mathrm{a}}, Q_{\mathrm{b}}$ and $Q_{\mathrm{s}}$ corresponding respectively to the antisymmetric stretching, the bending and the symmetric stretching normal modes of the linear molecule. In this first evaluation as well as in the following ones the transition dipole moment was determined using an original DIM like approach [15].

In a second step, instead of considering continuously the geometries $Q$, a sampling has been performed and a Wigner function weight used for $W_{i}(Q)^{2}$. In that case the moments are not free parameters but are selected according to the photon energy. The relation between $E$ and $Q$ is no longer univoque and a sampling has to be performed for each photon energy. It is also possible to perform a sampling from one (or various) classical trajectories in the ground state potential energy surface, but this restricts the selection to the classically allowed geometries and the resulting weights presents maxima at the turning points, a correct behaviour for hot molecules but a wrong one at low temperature.

In a third step, the continuum wave function was better taken into account assuming for both the ground and the excited state a Hartree product of 10 vibrational wave functions. The resulting spectrum corresponds to the convolution of the $1 \mathrm{D}$ spectrum [15]. Finally an exact 3D full quantum calculation of the visible spectrum has been performed using a time dependent method [20].

Interestingly, for $\mathrm{Ar}_{3}^{+}$all these approaches lead to similar spectra in good agreement with the experiments (see Fig. 1). This allowed a clear assignment of the visible spectrum. Two transitions contribute significantly. The main peak centered around $520 \mathrm{~mm}$ corresponds to a $\sum_{u^{+}} \rightarrow \sum_{g^{+}}\left(\right.$in $\left.D_{\infty h}\right)$ transition, it is a wide $(80 \mathrm{~nm})$ and intense feature $\left(\cong 10^{-16} \mathrm{~cm}^{2}\right)$ in good agreement with the experimental result. The absolute intensity is overestimated by the calculation by a factor of about 3 .

In a simple physical picture, this transition produces a nodal pattern to appear in the electronic charge distribution wave function. It presents thus some similarities with a colorcenter excitation which is dominated by an $s \rightarrow p$ transition. This transition is allowed for any distortion of the molecule and the variations of the dipole moment plays here a minor role. The 1D symmetric stretching spectrum alone is similar to the exact spectrum, an indication that this is the dominant motion during the short time dynamics which controls the shape of the main peak of the visible spectrum.

The second peak appears as a red shoulder centered around $600 \mathrm{~nm}$ and has a much lower 
intensity. According to our calculation $[15,17]$, it is due to a $\sum_{u^{+}} \rightarrow \Pi_{u}$ (in $\mathcal{D}_{\infty}$ ) transition which is forbidden for the linear symmetric geometry of the $\mathrm{Ar}_{3}^{+}$molecule but which becomes allowed mainly through bending distortions. The corresponding transition dipole moment increases strongly with the bending distortions and the resulting spectrum is still rather intense.

A comparison between the experimental and the theoretical spectra is given in Figure 1. We notice that the theoretical spectrum corresponds to a temperature of $0 \mathrm{~K}$ since it involves only the ground vibrational state. We also notice that the DIM model used here predicts a linear symmetric molecule. As long as one uses the quantum vibrational wave function with its width along each coordinate, it is not necessary to invoke neither a thermal broadening nor an asymmetric geometry for the molecule or a floppy molecule. Using the DIM model for the electronic Hamiltonian and the transition moment we get a good agreement with the experimental visible spectrum for $\mathrm{Ar}_{3}^{+}$. Such approaches receive thus a solid validation.

The approximate approaches involving vertical transitions and samplings of the initial conditions also correctly account for the visible absorption spectrum, opening the way for confident calculations of the absorption spectrum for the larger rare gas ionic clusters. It should be noticed that the quantum vibrational wave function of the ground state has always been involved in our work at least through approximate estimations. This point is important in relation with temperature effects, the width of the spectrum due to the intrinsic extension of the quantum wave function is reproduced by classical samplings at the expense of a temperature broadening effect. Therefore classical samplings lead intrinsically to an overestimation of the temperature and to a biased "thermometer" when adjustments to the experimental results are intended.

\subsection{UV Spectrum. - Following our DIM approaches for both the electronic Hamiltonian} and the dipole moment operators, the UV absorption spectrum corresponds to a transition to the highest state of $A^{\prime}$ symmetry, $\sum_{u^{+}} \rightarrow \sum_{u^{+}}$(in $\mathcal{D}_{\infty \mathrm{h}}$ ). This transition is forbidden at the equilibrium geometry of the molecule but is strongly enhanced by bending and antisymetric stretching distortions. Along the antisymmetric stretching distortions we obtain a discrete absorption spectrum with largely spaced lines $\left(2 \omega=1094 \mathrm{~cm}^{-1}\right.$ ), while the spectrum corresponding to the bending alone is continuous and its intensity strongly increases as excited vibrational levels of the ground state are involved.

The convolution of this two $1 \mathrm{D}$ spectra leads to the spectrum of Figure 2 where a temperature of $40 \mathrm{~K}$ has been used. This spectrum presents structures related to the antisymmetric stretching vibration. However, this spectrum is much less intense (by an order of magnitude) than the experimental results of DeLuca et al. [12] which is moreover structureless. These discrepancies can be related to temperature effects, since it is clear that the UV spectrum will be more intense for larger distortions on the bending and the antisymmetric stretching, the transition dipole moment increasing rapidly with this distortions. Another factor which will also contribute to broaden the structures is the averaging due to the symmetric stretching motions. It would be very interesting to check experimentally whether the absolute intensity significantly decreases with the temperature as predicted here or not.

\section{Photodissociation Dynamics}

An important question concerning the photodissociation of $\mathrm{Ar}_{3}^{+}$excited in the main visible absorption band is: why did the cluster dissociate into $\mathrm{Ar}^{+}[3-16]$ instead of into the lowest dissociative channel which is $\mathrm{Ar}_{2}^{+}$? In an attempt to answer this question and more generally to compute the branching ratio of the photofragments, one should investigate the long time dynamics of the system. In addition, since the systems starts with an excited electronic state 


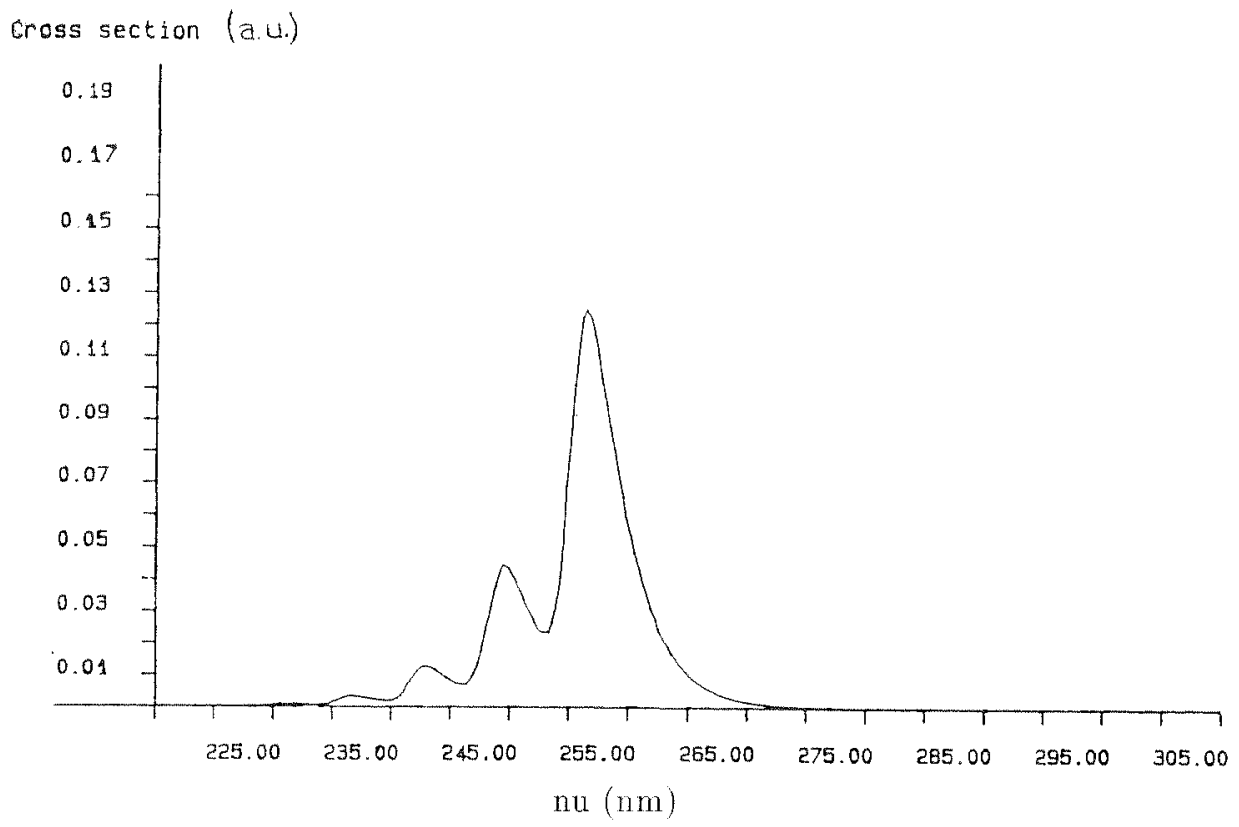

Fig. 2. - UV absorption spectrum resulting from a convolution of bending and antisymmetric stretching spectra.

and may undergo electronic state transitions during the decay, one should take into account the possible nonadiabatic transitions.

For that purpose the Hemiquantal dynamics with the Whole DIM (HWD) method was proposed [31] and developped [14-18]. It allows to treat all the possible electronic states together with a classical trajectory for the nuclei. The difference with a classical trajectory approach is that we do not compute first the potential, the derivatives and eventually the nonadiabatic couplings, but propagate directly both an electronic wave function spanned by the whole electronic basis set and a trajectory. We notice that the use of a DIM basis set is not a critical point, the main point is to use the complete basis set involved for the electronic Hamiltonian instead of some selected potential energy surfaces. The main interesting features of our approach arises from the use in the propagation of the whole representation of the electronic Hamiltonian operator, let us call it a potential Hamiltonian in contrast with a potential energy surface. Such a potential Hamiltonian should satisfy the dynamical treatment requirements: fiability, rapid computation for any geometry and availables analytical derivatives. The DNM approach offers an attractive opportunity in that context. It can however be replace by a different modelling for the electronic Hamiltonian. Within the HWD method, the expectation value of the time dependent electronic wave function defines an effective potential energy surface for the nucleus trajectory. The propagation of both the electronic wave vector and the trajectory do not need any a priori knowledge, neither on the potential energy surfaces nor on their nonadiabatic couplings. For the initial wave function, we often choose an eigenvector of the electronic Hamiltonian, in which case the trajectory starts on a given adiabatic potential energy surface.

Our experience shows that there are two phases in the theoretical study. During the first one some specific trajectories are run and analyzed in order to acquire the main physical infor- 
mations, during the second one statistical averages are performed in order to get quantitative specific results.

4.1. Initial Qualitative STUDY. The trajectories to be run and analyzed are selected from physical considerations. Here, for the photofragmentation study of $\mathrm{Ar}_{3}^{+}$, we started with geometries close to equilibrium on the surfaces which present a strong transition dipole moment, namely the second and third excited state of $\mathrm{A}^{\prime}$ symmetry (symmetric with respect to the molecular plane). This first phase gives the main physical insight on the system under study. The photoexcited $\mathrm{Ar}_{3}^{+}$ions rapidly dissociate in the $\mathrm{Ar}^{+}+\mathrm{Ar}+\mathrm{Ar}$ channel, mainly along a symmetric stretching motion. Looking for specific geometries which could allow for an $\mathrm{Ar}_{2}^{+}+$Ar dissociation, we found that only strongly bent molecules starting from the first excited PES of A' symmetry dissociate in that lowest channel. An inspection of the adiabatic potential energy surfaces reveals a conical intersection between the ground and the first excited PES around the equilateral geometries. Strongly bent distortions clearly helps in order to reach these configurations and to have a chance to perform a nonadiabatic transition to the ground state in order to dissociate into the $\mathrm{Ar}_{2}^{+}+\mathrm{Ar}$ channel.

The dominant symmetric stretching motion is related to the large gradient along this direction and very simply explains the observed bimodal kinetic energy distribution of the photo fragments. The central atom remains with a nearly zero kinetic energy during the dissociation while the onter ones transform in kinetic energy the excitation energy absorbed by the $\mathrm{Ar}_{3}^{+}$ molecule. The apparition of the centrai atom as a charged species deserves special attention. When the visible photon is absorbed in the main peak, the central atom carries no charge due to the $\sum_{\mathrm{g}}$ - character of the electronic wave function which has the largest transition dipole

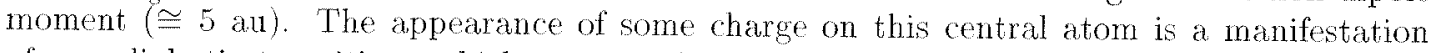
of nonadiabatic transitions which occur rather late during the dissociation according to our calculation (typically after $100 \mathrm{fs}$ ).

From only a few trajectories, we get a good insight on the photofragmentation dynamics of the $\mathrm{Ar}_{3}^{+}$ion. A sketch of this process is given in Figure 3. The molecule in the ground state is linear symmetric with nearly half the charge on the central atom $(1 / 4,1 / 2,1 / 4)$. A visible photom is absorbed, leading to a new electronic charge distribution, there is now nearly no charge on the central atom $(1 / 2,0,1 / 2)$ and large forces appear mainly along the symmetric stretching motion. The molecule explodes and only later on, as all PES become degenerate, some charge appears on the central atom.

4.2. Specifio Quantitative STudy. The first phase brings an interesting qualitative understanding but a quantitative study requires a statistical average over thousands of trajectories. That is the objective of the second phase. We actually adress a specific observable. for example the kinetic energy distribution of the photofragments, another observable could have been their branching ratio.

The main problem is now the sampling of the initial conditions. We should have in mind that the HWD method requires not only initial conditions for the geometries and the velocities (the classical variables) but also for the electronic wave function (the quantum variables). Various possibilities can be considered. We choose to start from a given adiabatic PES, the initial electronic wave function is thus nuambiguously determined. For example, for the photofragmentation study of $\mathrm{Ar}_{3}^{+}$, the starting state is assumed to be the third excited state of $\mathrm{A}^{\prime}$ symmetry (the one of $\sum_{\mathrm{g}}+$ symmetry for the equilibrium geometry), for the branching ratio study it should be taken as the second one (which has a $\Pi_{L}$ symmetry at the equilibrium geometry). This choice is guided by physical considerations. The knowledge of the transition dipole moment and the expected behaviour of the trajectory have been here determinant. We are not 
a) Ground state + photon

b) excited state $(t=0)$

c) dissociation

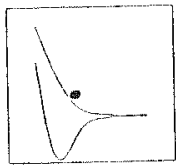

d) non adiabatic transition $(t \equiv 100 \mathrm{fs})$

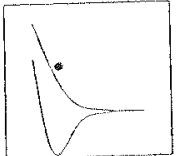

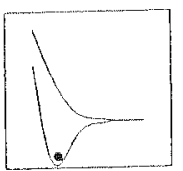
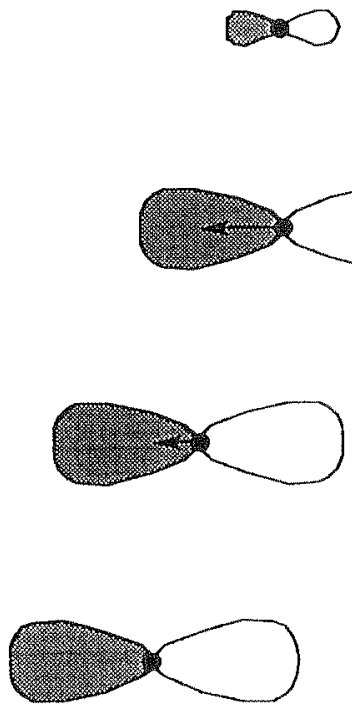
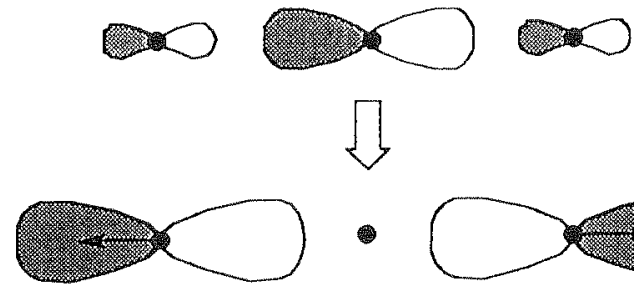

5

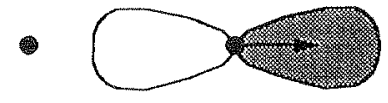

(9)
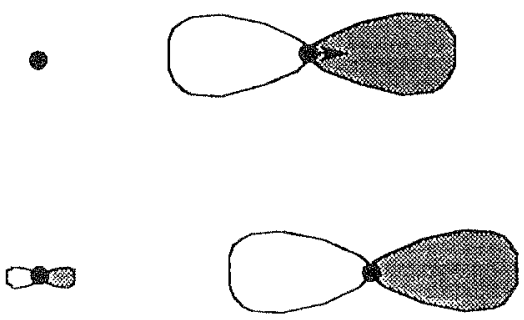

Fig. 3. Qualitative picture for the photofragmentation. The size of the p orbital illustrates the charge on the corresponding atom: a) the molecule is the ground state and has a nearly $\sum_{u+}$ character; b) a visible photon is absorbed, the molecule is now in the excited state of neatly $\sum_{g}+$ character, its the starting time for the fragmentation dynamics $(t=0)$, large repulsive forces appears for the two outer atoms; $c)$ the molecule is exploding and the outer atoms increase their kinetic energy; d) some charge appears on the central atom $(t \cong 100 \mathrm{fs})$.

constrain to stast from an adiabatic state, any normalized initial wave function is technically acceptable. To start with an adiabatic state is a convenient and appealing choice, and up to now we have adopted it.

For the classical variables the usual samplings of molecular dynamics can be used either with a fixed energy: sampling from trajectories running on the ground state, stochastic sampling within a domain, or with a fixed temperature: Monte Carlo or Nose samplings, Bolzman weights... Taking benefice of the knowledge of an approximate quantum vibrational wave function (Hartree product of 1D wave functions), we have adopted a Wigner like sampling where the trajectories are weighted according to their importance in phase space for the ground state. This procedure presents the advantages to take into account the classically forbidden configurations, to follow the quantum vibrational wave function and to control the energy absorbed by the molecule which is fixed by the photon energy. Thousands of HWD trajectories are then run and analysed according to the desired observable. To get the kinetic energy distribution, the linetic energy is discretized in small intervals and the atomic fragments having the corresponding energy comptabilized. The contribution of each fragment is proportional to its final charge and its initials Wigner weight and absorption intensity factors.

The final converged result (the convergence requires here about 5000 trajectories) for an excitation at $514 \mathrm{~mm}$ is shown in Figure 4. As expected, there are two peaks, one at zero kinetic energy corresponding exclusively to the central atom, the other at high kinetic energy corresponding to the two outer atoms. The sign of the velocity has not been taken into account, therefore the two outer atoms contribute to the same kinetic energy peak. However it is clear 


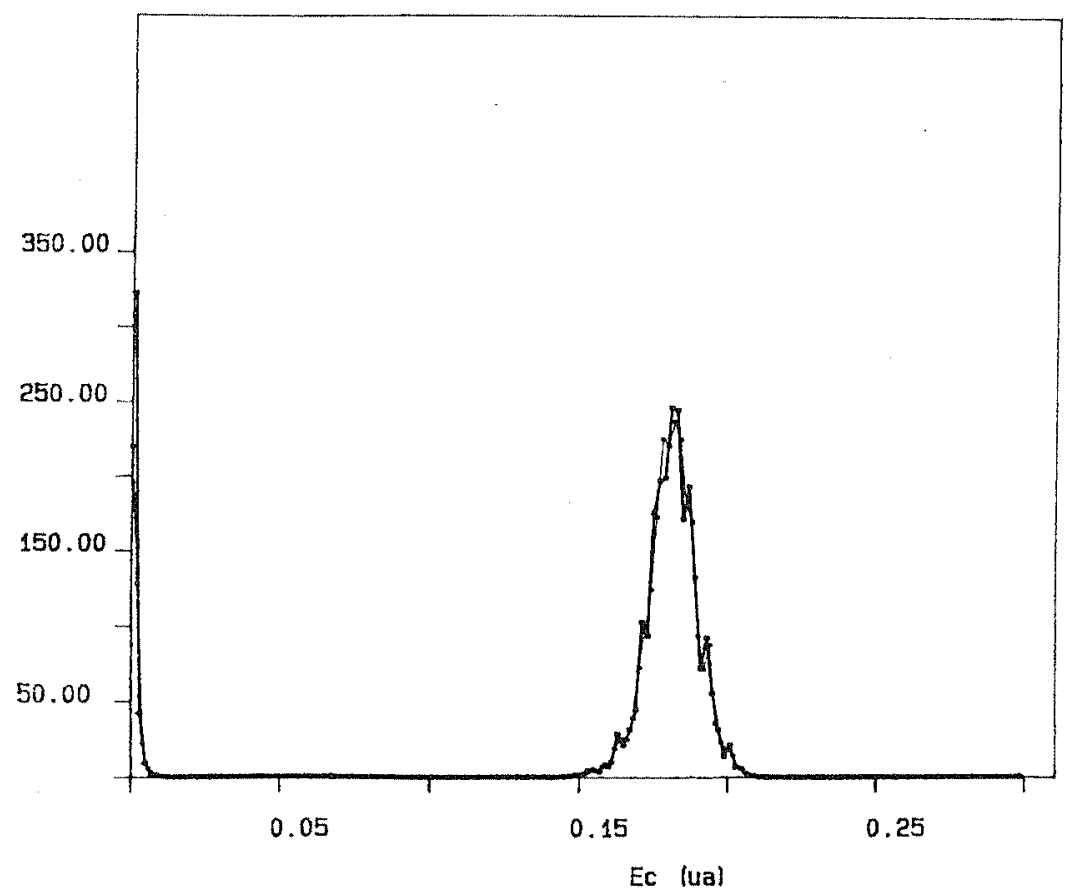

Fig. 4. - Kinetic energy distribution of $\mathrm{Ar}^{+}$photofragments, the photon energy is $514 \mathrm{~nm}$.

that their velocities have opposite signs and will lead to a three peak time of flight simulated spectrum. The relative ratio of the peaks area is $15 \%$ for the slow $\mathrm{Ar}^{+}$and $85 \%$ for the fast ones. It is in reasonable agreement with the experimental result: $19 \%$ slow, $81 \%$ fast.

\section{Conclusion}

The agreement of the structural results for $\mathrm{Ar}_{3}^{+}$with the best ab initio calculations together with the reasonable agreement of the calculated absorption spectra, the fragmentation ratio and the kinetic energy distribution with the well established experimental results, validates our approach for realistic general studies of the larger positive ions of rare gas clusters. This approach is based on a DIM like modelling for both the electronic Hamiltonian and the dipole moment operators, on approximate methods for the absorption spectrum estimation, and on the HWD method together with adequate samplings of the initial conditions. This approach allows for realistic dynamical simulations in both the short time scale (photoabsorption spectrum) and the long time scale (nonadiabatic transitions, dissociation, fragmentation, decay,...). We have shown that, on one hand, from a preliminary study qualitative but detailed understandings on the system can be acquired, and on the other hand that quantitatives results can be obtained from statistical averages. Both studies present an interesting predictive character since they are based on effective integrals extracted from $a b$-initio calculations and require no a priori knowledge on the potential energy surfaces or on the dynamics of the system.

For the heavier rare gases, the spin orbit coupling is expected to play an important role for the structural aspects as well as for the short and long time scale dynamics. Work is in progress in order to generalized our approach in order to include this spin orbit interactions. 


\section{Acknowledgments}

We gratefully thank. J.A. Beswick for his critical reading of the manuscript and acknowledge the CNRS / DRU convention for supports during the visits of M. A. in Toulouse.

\section{References}

[1] Physics and Chemistry of Finite Systems: From Clusters to Crystals, P. Jena, S.N. Khanna and B.K. Rao Eds., NATO ASI, Set. C, vol 174 (Proceedings of Richmond).

[2] Proceedings of the fifth International Meeting on Small Particles and Inorganic Clusters, Z. Phys. D 20 (1990).

[3] Albertoni C.R., Kuher R., Sarkis H.V. and Castelman Jr A.W., J. Chem. Phys. 87 (1987) 5043.

[4] Levinger N.E., Ray D., Murray K.K., Mullin A.S., Schulz C.P. and Lineberger W.C., J. Chem. Phys. 89 (1988) 71.

[5] Chen Z.Y., Albertoni C.R., Hasegawa M., Khun R. and Castelman Jr A.W., J. Chem. Phys. 91 (1989) 4019.

[6] Levinger N.E., Ray D., Alexander M.L. and Lineberger W.C., J. Chem. Phys. 89 (1988) 5654.

[7] Woodward C.A., Upham J.E., Stace A.J. and Murrel J.N., J. Chem. Phys. 91 (1989) 7612.

[8] Nagata T., Hirokawa J. and Kondow T., Chem. Phys. Lett. 176 (1991) 526.

[9] Nagata T., Hirokawa J., Ikegami T., Kondow 'T. and Iwata S., Chem. Phys. Lett. 180 (1991) 433.

[10] Bowers M.T., Palke W.E., Robins K., Roehl C. and Walsh S., Chem. Phys. Lett. 180 (1991) 235.

[11] Gotts N.G., Hallet R., Smith J.A. and Stace A.J., Chem. Phys. Lett. 181 (1991) 491.

[12] DeLuca M.J. and Johnson M.A., Chem. Phy.s. Lett. 162 (1989) 445.

[13] Haberland H., Von Issendorff B., Kolar T., Kornmeir H., Ludewigt C. and Rish A., Phys. Rev. Lett. 67 (1991) 3290.

[14] Amarouche M., Gadea F.X. and Durup J., Chem. Phys. 130 (1989) 145.

[15] Gadea F.X. and Amarouche M., Chem. Phys. 140 (1990) 385.

[16] Cadea F.X. and Durup J., Chem. Phys. Lett. 181 (1991) 378.

[17] Gadea F.X., Z. Phys. D 20 (1991) 25.

[18] Gadea F.X. and Durup J., Laser Chem. 11 (1991) 95.

[19] Gadea F.X. Svarda I. and Paidarova I., Chem. Phys. Lett. 223 (1994) 369.

[20] Gadea. F.X. and Le Quéré F., J. Chem. Phys. 102 (1995) 7830.

[21] Hartke B., Gibson D.A. and Carter E., Int. J. Quant. Chem. 45 (1993) 59.

[22] Deunens E., Diz A. Taylor H. and Ohrn Y., J. Chem. Phys. 96 (1992) 6820.

[23] Pastore G., Smargiassi E. and Buda F., Phys. Rev. A 44 (1991) 6334.

[24] Gadea F.X. and Kuntz P.J., Mol. Phys. 63 (1988) 27.

[25] Hesslich J. and Kuntz P.J., Z. Phys. D 2 (1986) 251.

[26] Kuntz P.J. and Valdorf J., Z. Phys. D 8 (1988) 195.

[27] Wadt W.R., Appl. Phys. Lett. 38 (1981) 1030.

[28] Bohmer H.U. and Peyerimhof S.D., Z. Phys. D 3 (1986) 195.

[29] Chen Z.Y., May B.D. and Castelman J. A.W., Z. Phys. D 25 (1993) 239.

[30] Last I. and George T.F., J. Chem. Phys. 93 (1990) 8925.

[31] Gadea. F.X., Thèse d'Etat, Toulouse, France (1.987).

[32] Amarouche M. Thèse de l'Université Paul Sabatier (1988). 